\title{
NEUROPROTECTION WITH MELATONIN AND THE CASPASE-2 INHIBITOR TRP601: NOVEL ASPECTS BY ANALYSIS OF THE METABOLOME IN EXCITOTOXIC BRAIN INJURY
}

\author{
M. Keller ${ }^{1}$, D. Enot ${ }^{1}$, M. Hodson ${ }^{2}$, L. Schwendimann ${ }^{3}$, P. Gressens ${ }^{3}$ \\ ${ }^{I}$ Department of Pediatrics, University Hospital Essen, Essen, Germany, ${ }^{2}$ BIOCRATES Life Sciences AG, \\ Innsbruck, Austria, ${ }^{3}$ INSERM, Paris, France
}

Background and aims: Developmental brain injury in the preterm infant is a serious condition with subsequent long-term neurological morbidity. Promising therapeutic candidates are Melatonine and the caspase-2 inhibitor,TRP601. Their mode of action is not yet fully elucidated. In this study we aim to evaluate potential metabolic mechanisms in excitotoxic brain injury.

Methods: 150 mice pups received at P5 two i.c. injection of ibotenic acid. Animals were randomized at P6 to receive i.p. bolus injection of saline-vehicle, melatonine $(0.5 \mathrm{mg} / \mathrm{kg})$ or TRP601 $(1 \mathrm{mg} / \mathrm{kg})$. Brain and plasma samples were collected at P6, P7, P8, P10 and P30 and subjected to the quantitation of 230 metabolites. Data modelling was adapted to address concentration changes at various levels (metabolite, chemical class and biochemical pathway) in plasma, brain and at the interface of both.

Results: Brain and plasma metabolomes are clearly affected after the intervention and drug administration including at P30. Biostatistic functional analysis highlighted commonalities (e.g., prostanoids and sphingomyelins respectively down and up-regulated), discrepancies (e.g., strong suppression of inflammation markers after melatonine injection) and different dynamics in the response to the two drugs as highlighted by delayed triggering of citric acid cycle in TRP601 (P10) compared to Melatonine (P8).

Conclusions: We show that combining comprehensive metabolite profiling with multilevel statistical analysis offers a level of granularity that could serve as a basis for optimising neuroprotection strategy and timing in/without combination with other therapeutic protocols. Since some of these changes were also reflected in plasma this also enables biomarker discovery for theranostics. 